Original Article

\title{
The recovery time required for rat joint contractures treated with joint fixation with unweighting of the hind limbs
}

\author{
Yuta Sato, RPT, PhD ${ }^{1)^{*}}$, TAKeya Ono, RPT, PhD ${ }^{1)}$, Hideki Ishikura, RPT, PhD ${ }^{2)}$, \\ Kazuki Aihara, RPT, PhD ${ }^{3)}$, Atsushi Tasaka, RPT, PhD $^{3)}$, Namiko Umei, RPT, PhD $^{1)}$, \\ Wakako TsumiYama, RPT, $\mathrm{PhD}^{1)}$, SadaAki OKi, MD, $\mathrm{PhD}^{1)}$ \\ 1) Department of Physical Therapy, Faculty of Health and Welfare, Prefectural University of Hiroshima: \\ 1-1 Gakuen-cho, Mihara City, Hiroshima 723-0053, Japan \\ 2) Department of Rehabilitation, Faculty of Health Sciences, Hiroshima Cosmopolitan University, Japan \\ 3) Department of Rehabilitation Science, Osaka Health Science University, Japan
}

\begin{abstract}
Purpose] The purpose of this study was to investigate whether the recovery period of joint contractures differ between treatments with joint fixation with unweighting of the hind limbs and joint fixation alone. [Subjects and Methods] Twelve 10-week-old male Wistar rats were divided into a group with joint fixation of the right ankle joints in full plantar flexion for 1 week (F) and a group with joint fixation and hind limb suspension (FS). Thereafter, the rats were removed from the joint fixation and hind limb suspension for 2 weeks. The ankle dorsiflexion angles during the recovery process of the joint contractures were measured. [Results] The ankle dorsiflexion angles of the F group from the day joint fixation was removed to the seventh recovery day were significantly lower than the pre-experiment values. The ankle dorsiflexion angles of the FS group from the day joint fixation and hind limb suspension were removed to the 10th recovery day were significantly lower than the pre-experiment values. [Conclusion] We found that the period required for the recovery of joint contractures treated with joint fixation with unweighting of the hind limbs was longer than that with joint fixation alone.

Key words: Joint contracture, Recovery, Hind limb suspension
\end{abstract}

(This article was submitted Nov. 15, 2018, and was accepted Jan. 3, 2019)

\section{INTRODUCTION}

The definition of joint contracture is a limitation in joint range of motion (ROM) derived from an organic change in the periaticular soft tissues, such as skeletal muscle, joint capsule and skin ${ }^{1}$. A patient with a joint contracture may show an increase in the possibility of falling ${ }^{2}$ and many difficulties in daily life ${ }^{3,4)}$. Therefore, the treatment of joint contracture is important.

Yaoita found that the period required for the recovery of a knee joint contracture caused by joint fixation in 90 degrees of flexion is $10-70$ days $^{5)}$. As a result, it was shown that the period required for the recovery of a joint contracture gets longer with the prolongation of the period of joint fixation. Furthermore, it was found that when joint fixation continues for more than 30 days the total recovery of a joint contracture to normal is unattainable. These results indicate that the treatment of a joint contracture gradually becomes more difficult with the prolongation of a joint contracture. Therefore, it is important that the treatment of a joint contracture begins at an early phase.

In addition, we studied the effect of a joint contracture caused by joint fixation in full plantar-flexion ${ }^{6-17)}$ and hind limb suspension reproduced by unweighting the hind limbs ${ }^{13-20)}$ for one week $^{13-17)}$.

*Corresponding author. Yuta Sato (E-mail: y-satou@pu-hiroshima.ac.jp)

(C2019 The Society of Physical Therapy Science. Published by IPEC Inc.

(c) (1) $\odot$ This is an open-access article distributed under the terms of the Creative Commons Attribution Non-Commercial No Deriva-

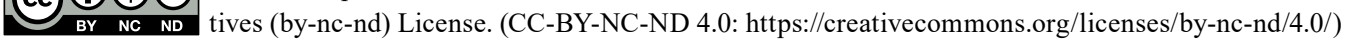


As a result, it was found that a joint contracture caused by joint fixation with unweighting the hind limbs has a severer effect than that caused by joint fixation alone. These results indicate that unweighting the hind limbs facilitates an exacerbation of the joint contracture. Therefore, it was considered that the period required for recovery of a joint contracture caused by joint fixation with unweighting of the hind limbs was more prolonged than that caused by joint fixation for the same period. In the previous study), it was shown that the period required for the recovery of a joint contracture depend on the period of joint fixation. Therefore, if the period of joint fixation was the same, unweighting the hind limbs may not affect the period required for the recovery of a joint contracture. But, no study investigating the period required for the recovery of a joint contracture caused by joint fixation with unweighting of the hind limbs has been done.

The purpose of this study was to investigate whether there is a difference in the period required for recovery of a joint contracture caused by joint fixation with unweighting the hind limbs as compared by joint fixation alone.

\section{SUBJECTS AND METHODS}

The subjects were twelve 10-weeks-old male Wistar rats. All rats were housed in individual cages and were given free access to water and standard rat food. The room temperature at $23{ }^{\circ} \mathrm{C}$ was controlled by an air conditioner. In addition, the day-night cycles were artificially regulated by setting lights-on for $12 \mathrm{~h}$ and lights-off for $12 \mathrm{~h}$ every day. This study was approved by the Animal Experiments Committee of the Prefectural University of Hiroshima and performed according to the University Regulations on Animal Experiments (Approval number: 13MA009). All treatments, such as joint fixation, hind limb suspension, and measurement of joint ROM, were performed under anesthesia.

The period of this study was three weeks. All rats were divided into the group for the right ankle joint fixation in full plantar-flexion $(\mathrm{F}, \mathrm{n}=6)$ or the group for joint fixation and hind limb suspension $(\mathrm{FS}, \mathrm{n}=6)$.

The right hind limbs of all rats were fixed in full plantar-flexion by using non-elastic tape. All the right toes were exposed for the confirmation of the presence or absence of edema. The protection covering the joint fixation was made with a wire mesh. The rewinding of the non-elastic tape was performed as appropriate when edema or a loosened tape was found.

For the hind limb suspension of the FS group, a 1.0 diameter Kirchner wire was inserted into the rat's tail to form a triangle and then combined with a swivel hook. The hind limb suspension was performed by hanging the swivel hook to the horizontal bar at the top of cage. Thus, the rats' hind limbs were set in an unweighted state. However, as the pain caused by the insertion of the Kirchner wire might affect the joint contracture, the Kirschner wire was also inserted into the rat's tail of the $\mathrm{F}$ group. The rats were allowed $360^{\circ}$ rotation by a swivel hook, and free access to standard food and water was possible by using their fore limbs. The joint fixation and hind limb suspension were performed for one week. Thereafter, the rats were removed from the joint fixation and hind limb suspension for two weeks.

The right ankle dorsiflexion angles of all rats were measured pre-experiment (Pre), after one week of joint fixation and hind limb suspension (1w), and every day after joint fixation and hind limb suspension had been removed (RD1-14). For the measurements of ankle joint ROM, the rat was positioned on its side with the hip and knee joint fixed in flexion by using the flexion equipment as described in our previous study ${ }^{21)}$. The rat's fibula head and lateral malleolus were marked for the measurement of the ankle joint ROM. A force of $0.3 \mathrm{~N}$ was applied by using a tension meter (LTS-1KA; Kyowa Electronic Instruments Co., Japan) perpendicular to the sole of the foot for passive dorsiflexion.

This dorsiflexion force of $0.3 \mathrm{~N}$ is the minimum force that is enough to move a normal rat's ankle joint into the full dorsiflexion position ${ }^{6}$. While the right ankle joint was passively dorsiflexed, the ankle joint movement was recorded from directly above the hind limb using a digital camera. The recorded videos were downloaded to a computer to extract still images of dorsiflexion at the moment of application of the $0.3 \mathrm{~N}$ force. The ankle dorsiflexion angles were measured from the still images using computer software (ImageJ version $1.48 \mathrm{v}$; USA). The definition of ankle dorsiflexion angle is the angle obtained from a line connecting the head of fibula and lateral malleolus and a line parallel to the bottom of the heel to eliminate forefoot movement from the measurement. The ankle dorsiflexion angles were measured 3 times, and the mean value was used in the analysis.

All statistical analyses were performed by using the BellCurve for Excel ver. 2.02 (Social Survey Research Information Co., Ltd., Tokyo, Japan). In this study, statistical analysis methods were chosen to investigate the temporal change of ankle dorsiflexion angles in the F and FS groups. For each period of measurement of the ankle dorsiflexion angles in all groups compared with the Pre, the repeated one-way ANOVA or Friedman test was performed corresponding to the presence or absence of normality after using the Shapiro-Wilk test. In the case of a presence of normality, the Dunnett Test or Steel Test was performed for the post hoc tests. A significant difference was concluded to exist at a probability value of less than $5 \%$ in all of the statistical tests.

\section{RESULTS}

The means and standard deviations of the ankle dorsiflexion angles of each group for each period of measurement are shown in Table 1. For the ankle dorsiflexion angles of the F group, the Friedman Test and Steel Test were performed because there was an absence of normality found. As a result of the statistical analyses, the significant difference in the F group when compared with Pre were confirmed from 1w to RD7. However, a repeated one-way ANOVA and Dunnett Test were 
Table 1. The change in the ankle dorsiflexion angle (in degrees)

\begin{tabular}{ccccc}
\hline Evaluation time & F group & \multicolumn{3}{c}{ FS group } \\
\hline Pre & $130.7 \pm 2.3$ & $*$ & $131.6 \pm 3.9$ & $*$ \\
1w & $95.4 \pm 5.8$ & $*$ & $69.9 \pm 10.0$ & $*$ \\
RD1 & $105.2 \pm 7.6$ & $*$ & $85.0 \pm 8.0$ & $*$ \\
RD2 & $104.0 \pm 8.7$ & $*$ & $91.5 \pm 8.3$ & $*$ \\
RD3 & $108.6 \pm 7.9$ & $*$ & $97.0 \pm 7.5$ & $*$ \\
RD4 & $110.8 \pm 6.7$ & $*$ & $95.7 \pm 4.9$ & $*$ \\
RD5 & $112.9 \pm 6.1$ & $*$ & $97.0 \pm 8.5$ & $*$ \\
RD6 & $118.8 \pm 2.5$ & $*$ & $100.4 \pm 7.7$ & $*$ \\
RD7 & $115.1 \pm 7.9$ & $*$ & $105.7 \pm 12.6$ & $*$ \\
RD8 & $121.4 \pm 4.0$ & & $109.1 \pm 8.1$ & $*$ \\
RD9 & $122.9 \pm 6.3$ & & $111.0 \pm 10.0$ & $*$ \\
RD10 & $123.7 \pm 6.1$ & & $112.8 \pm 7.6$ & $*$ \\
RD11 & $127.8 \pm 2.6$ & & $118.3 \pm 14.8$ & \\
RD12 & $125.8 \pm 4.5$ & & $119.0 \pm 11.5$ & \\
RD13 & $121.8 \pm 3.4$ & & $121.5 \pm 9.1$ & \\
RD14 & $126.0 \pm 4.7$ & & $119.3 \pm 7.5$ & \\
\hline All
\end{tabular}

All values are shown as mean \pm SD.

Pre: Pre-experiment; 1w: One week after starting the experiment; RD1-14: The recovery days after ending joint fixation and hind limb suspension.

*Significant change as compared with Pre $(\mathrm{p}<0.05)$.

performed on the ankle dorsiflexion angles for the FS group because a presence of normality was found. As a result of the statistical analyses, the significant difference in the FS group as compared with Pre were confirmed from 1w to RD10.

\section{DISCUSSION}

Joint contracture is caused by joint fixation ${ }^{5-17)}$, and it exacerbates when combined with unweighting the hind limbs ${ }^{13-16)}$. The decreased skeletal muscle extensibility ${ }^{7,8,15)}$ is the main factor in a joint contracture caused by joint fixation for one week, and for the exacerbation caused when combined with the unweighting of the hind limbs ${ }^{14,15)}$. In this study, the ankle dorsiflexion angles in both groups at $1 \mathrm{w}$ were more significantly lowered than that at Pre. Therefore, we considered that the cause of the joint contractures in both groups might be the skeletal muscle, and the extensibility in the FS group might be lower than that in the F group. The stretching of skeletal muscle may improve its extensibility ${ }^{9}$. The normal rat's ankle joint moves widely from the plantar-flexion position to the dorsiflexion position ${ }^{18}$. Therefore, we deduced that the rat's skeletal muscles around the ankle joint were stretched by the active movement and weighting of the hindlimbs at $1 \mathrm{w}$ to RD14 in this study. It was considered that this effect of stretching of the skeletal muscle was the main factor that facilitated the healing of the joint contractures in both groups.

The period required for recovery of joint contracture caused by joint fixation for one week is one week ${ }^{10)}$. In this study, the period required for the recovery of the ankle dorsiflexion angle in the F group was one week. However, there is no known study on the period required for the recovery of a joint contracture caused by joint fixation with unweighting of the hind limbs. There is one study in which the ankle dorsiflexion angle was measured after one week of joint fixation and hind limb suspension was removed ${ }^{17}$. According to the study, the ankle dorsiflexion angle did not recover to the same state as was measured in the pre-experiment. On RD7 of this study, the ankle dorsiflexion angle in the FS group was even more significantly lower than that in Pre. Therefore, it was shown that the period required for recovery of a joint contracture caused by joint fixation with unweighting the hind limbs is longer than that caused by joint fixation alone.

It is considered that the decreased skeletal muscle extensibility caused by joint fixation is induced by an increase in skeletal muscle collagen ${ }^{1,11,12,22)}$. On the other hand, since the skeletal muscle collagen in the joint contracture caused by the joint fixation with unweighting of the hind limbs was increased more than that caused by joint fixation alone ${ }^{16)}$, the skeletal muscle extensibility in the joint contracture caused by joint fixation with the unweighting of the hind limbs was lower than that caused by joint fixation alone ${ }^{14,15)}$. Furthermore, it was reported in previous study that the skeletal muscle collagen and the factor related to the increase in skeletal muscle collagen increased after the hindlimb suspension had been removed ${ }^{20)}$. It is considered that the increased skeletal muscle collagen caused by reweighting the hind limbs was the factor that inhibited the recovery of the joint contracture caused by joint fixation with the unweighting of the hind limbs. In this study, the recovery period of the joint contracture in the FS group might have been prolonged by an aggravating influence of reweighting after the hind limb suspension had been removed. 
A joint contracture gradually exacerbates following a prolongation of the period of joint fixation ${ }^{7)}$. Also, the period required for the recovery of a joint contracture gradually increases following a prolongation of the period of joint fixation ${ }^{5}$. Therefore, it is considered that the period required for the recovery of a joint contracture is related to the severity of the joint contracture.

Furthermore, if the period of joint fixation is over 30 days, the joint contracture recovers but only incompletely ${ }^{5)}$. In this study, the joint contractures in the FS group completely recovered to the Pre state. Therefore, it was clarified that a joint contracture caused by joint fixation with unweighting of the hind limbs for one week exacerbated more than that caused by joint fixation alone, but it was a reversible change.

The new finding obtained from this study is that the effects of a joint contracture caused by joint fixation with the unweighting of the hind limbs are more prolonged than that caused by joint fixation alone, but the recovery might be complete. The results of this study show that stretching by active movement and weighting the hind limbs for a joint contracture caused by joint fixation with unweighting the hind limbs are effective. Furthermore, these findings are very meaningful for comparing and examining better treatment methods for humans.

The limitation of this study is that the temporal change necessary for the recovery process of the skeletal muscle and collagen in a joint contracture was not clarified. We recommend that the temporal change for the skeletal muscle recovery process in a joint contracture be investigated in future studies.

\section{Conflict of interest}

There are no conflicts of interest in this study.

\section{REFERENCES}

1) Okita M, Sakamoto J, Honda Y, et al.: Limited range of joint motion—Mechanisms and treatment-2nd ed. Tokyo: Miwa-Shoten, 2013, pp 1-20, 93-134 (in Japanese).

2) Murata S, Tsuda A, Inatani F, et al.: Physical and cognitive factors associated with falls among the elderly with disability at home. J Jpn Phys Ther Assoc, 2005, 32: 88-95 (in Japanese).

3) Koizumi K, Ogawa A, Murayama K, et al.: Prevention and treatment of contracture. Tokyo: Igaku-Shoin, 2003, pp 1-17 (in Japanese)

4) Fukuya Y: The relation between motion of daily living and joint range of motion in the adult with central nervous disturbance. J Jpn Phys Ther Assoc, 1994, 21: 90-93 (in Japanese).

5) Yaoita M: [Experimental study on the genesis of knee joint immobilization induced by long-term fixation and its remobilization]. Nihon Seikeigeka Gakkai Zasshi, 1966, 40: 431-453 (in Japanese). [Medline]

6) Oki S, Otsuka A, Kanai S, et al.: The effects of range of motion exercise to prevent joint contracture in the rats. J Phys Med, 2004, 15: 312-316 (in Japanese).

7) Okamoto M, Okita M, Kasuya A, et al.: Effects of immobilization period on restriction of soft tissue and articulation in rat ankle joint. J Jpn Phys Ther Assoc, 2004, 31: 36-42 (in Japanese).

8) Ono T, Oki S, Umei N, et al.: Effect of an intermittent eight-hour joint fixation period on joint contractures in rats. J Phys Ther Sci, 2014, 26: 53-55. [Medline] [CrossRef]

9) Ishikura H, Ono T, Oki S, et al.: Effect of stretch on improvement of muscular contractures in rats. J Phys Ther Sci, 2015, 27: 2821-2823. [Medline] [CrossRef]

10) Takemoto Y, Fukuoka A, Oki S, et al.: The effect of joint contracture upon the position of joint fixation in neutral: an experimental study on the rat ankle. J Phys Med, 2010, 21: 255-259 (in Japanese).

11) Honda Y, Kondo Y, Yokoyama S, et al.: Fudokikan no entyo ni tomonau rat hiramekin no kinsyumaku narabini kinnaimaku ni okeru type I.III collagen no henka. Rigakuryohogaku, 2011, 38(Suppl. 2): PF2-016 (in Japanese).

12) Okita M, Nakano J, Sakamoto J, et al.: Itami to kosyuku: Kokkakukin no henka kara mita kosyuku no byotai. Nihon Undoki Totsu Kenkyukaishi, 2010, 2 : 31-38 (in Japanese).

13) Sato Y, Ono T, Ishikura H, et al.: Effect of unweighting the hind limbs of rats on joint contractures. Rigakuryoho Kagaku, 2015, 30: 409-412 (in Japanese). [CrossRef]

14) Sato $\mathrm{Y}$, Ono $\mathrm{T}$, Ishikura $\mathrm{H}$, et al.: Effects of rat hind limb unweighting on the extensibility of the soleus and skin around a joint contracture. Rigakuryoho Kagaku, 2016, 31: 755-758 (in Japanese). [CrossRef]

15) Sato Y, Ono T, Ishikura H, et al.: Study on change of rat soleus extensibility caused by reduction in joint movement with unweighting of the hind limbs. J Phys Ther Sci, 2018, 30: 53-57. [Medline] [CrossRef]

16) Matsumoto T, Ono T, Ishikura H, et al.: Effects of joint immobilization and hindlimb unloading on collagen fibers of soleus muscles in rats. J Phys Ther Sci, 2017, 29: 1192-1195. [Medline] [CrossRef]

17) Sato Y, Ono T, Ishikura H, et al.: Treadmill hoko ga kansetsukotei to koshikensui ni yotte syozita kansetsukosyuku ni oyobosu eikyo: rat wo mochiita zikkentekikenkyu. Rigakuryohogaku, 2016, 44 (Suppl. 2): P-KS-39-1 (in Japanese).

18) Riley DA, Slocum GR, Bain JL, et al.: Rat hindlimb unloading: soleus histochemistry, ultrastructure, and electromyography. J Appl Physiol, 1990, 69: 58-66. [Medline] [CrossRef]

19) Hirose T, Nakazato K, Song H, et al.: TGF- $\beta_{1}$ and TNF- $\alpha$ are involved in the transcription of type I collagen $\alpha_{2}$ gene in soleus muscle atrophied by mechanical unloading. J Appl Physiol, 2008, 104: 170-177. [Medline] [CrossRef]

20) Heinemeier KM, Olesen JL, Haddad F, et al.: Effect of unloading followed by reloading on expression of collagen and related growth factors in rat tendon and muscle. J Appl Physiol, 2009, 106: 178-186. [Medline] [CrossRef]

21) Tasaka A, Ono T, Oki S, et al.: The reliability of two ROM measurement methods for rats. Rigakuryoho Kagaku, 2014, 29: 143-145 (in Japanese). [CrossRef]

22) Okano A, Okita M, Okuno H, et al.: Kinosyogaikagakunyumon. Fukuoka: Kyushu Shinryobunko, pp 230-240 (in Japanese). 